\title{
ON NONLINEAR EVOLUTION OPERATORS ASSOCIATED WITH SOME NONLINEAR DISPERSIVE EQUATIONS
}

\author{
SHINNOSUKE OHARU ${ }^{1}$ AND TADAYASU TAKAHASHI
}

\begin{abstract}
The initial-boundary value problem for a nonlinear dispersive system with time-dependent boundary condition is discussed in the Sobolev space $H^{1}$ from the point of view of the theory of nonlinear evolution operators. A notion of weak solution to the problem is introduced and the associated family of solution operators is constructed in such a way that it gives rise to a nonlinear evolution operator with time-dependent domain. Various qualitative properties as well as regularity of the weak solutions are investigated through those of the constructed evolution operator.
\end{abstract}

Introduction. This paper is concerned with the initial-boundary value problem for the nonlinear dispersive equation

$$
\begin{array}{ll}
u_{t}+(\phi(u))_{x}-u_{x x t}=0, & 0<t<T, \quad 0<x<1, \\
u(0, x)=u_{0}(x), & 0 \leq x \leq 1, \\
u(t, 0)=h_{0}(t), u(t, 1)=h_{1}(t), & 0 \leq t \leq T,
\end{array}
$$

where $T>0, h_{i}, i=0,1$, are given functions of class $C^{1}[0, T] ; \phi$ is a nonlinear function of class $C^{1}(\mathbf{R})$ satisfying $\phi(0)=0$ and the growth condition

$$
\limsup _{|\xi| \rightarrow \infty}|\phi(\xi)| /|\xi|^{2}<\infty ;
$$

and the initial function $u_{0}$ is supposed to satisfy the compatibility condition $u_{0}(0)=$ $h_{0}(0), u_{0}(1)=h_{1}(0)$. Equation (1) is regarded as a mathematical model for long waves of small amplitude. A precise exposition on the derivation of equation (1) was given in [1] for the particular case $\phi(\xi)=\xi+\xi^{2} / 2$, and so far various problems related to (1) have been treated in $[\mathbf{2 , 3 , 6 , 9}$. In particular, the work of Showalter [10] contains a more general treatment of nonlinear dispersive systems. In the previous paper [6] we indicated that an operator-theoretic approach was efficient for the case in which (3) is replaced by the periodic boundary condition: $u(t, 0)=$ $u(t, 1), 0 \leq t \leq T$, and that the problem was minutely investigated in terms of nonlinear semigroup theory. Here we put the growth condition (4) on the nonlinear function $\phi$ and discuss the global solvability as well as various qualitative properties of solutions of (1) under the nonperiodic boundary condition (3). It is fairly easy to see that the problem (1)-(3) has a local (weak) solution even if condition (4) is not assumed. However, we do not know at this moment whether or not the problem

Received by the editors February 28, 1984 and, in revised form, May 5, 1985.

1980 Mathematics Subject Classification. Primary 58D25, 35Q20, 47H20; Secondary 35K22, $47 \mathrm{H} 17$.

Key words and phrases. Nonlinear dispersive equations, long waves of small amplitude, nonlinear evolution operators.

${ }^{1}$ Research supported by the Grant-in-Aid for Scientific Research from the Ministry of Education of Japan. 
admits a solution on all of the given interval $[0, T]$ without restrictions on $\phi$ such as (4).

In this note we convert under condition (4) the problem (1)-(3) into the Cauchy problem for a time-dependent evolution equation of the form

$$
u^{\prime}(t)=A(t) u(t), \quad 0<t<T,
$$

in the Sobolev space $H^{1}(0,1)$ and apply a recent result $[7]$ on time-dependent nonlinear evolution equations to obtain a nonlinear evolution operator $\mathfrak{U}$ in $H^{1}(0,1)$ which provides $C^{1}$-solutions of (5).

For each $t \in[0, T]$ we define a closed convex subset $K(t)$ of $H^{1}(0,1)$ by

$$
K(t)=\left\{v \in H^{1}(0,1): v(0)=h_{0}(t), v(1)=h_{1}(t)\right\} .
$$

We shall show (in Theorem 3.1) that the evolution operator $\mathfrak{U}$ is obtained as a family of nonlinear operators $\{U(t, s): 0 \leq s \leq t \leq T\}$ in $H^{1}(0,1)$ with the properties:

(E1) For $r, s, t \in[0, T]$ with $r \leq s \leq t$, the operator $U(t, s)$ maps $K(s)$ into $K(t)$, $U(s, s)=I$ on $K(s)$, and $U(t, s) U(s, r)=U(t, r)$ on $K(r)$.

(E2) For $s \in[0, T)$ and $z \in K(s)$, the $H^{1}(0,1)$-valued function $U(\cdot, s) z$ is continuously differentiable on $[s, T]$ and satisfies equation $(5)$ on $(s, T)$. Hence $u(t) \equiv U(t, 0) u_{0}$ gives a $C^{1}$-solution of (5) satisfying

$$
u(0)=u_{0} \in K(0) .
$$

Following [7], the evolution operator $\mathfrak{U}$ is said to be constrained in the family $\mathfrak{K}=\{K(t): t \in[0, T]\}$ and is understood to be defined on a noncylindrical domain $\bigcup_{0 \leq s \leq T}(\{s\} \times K(s))$ since $K(s) \cap K(t)=\varnothing$ if $h_{i}(s) \neq h_{i}(t)$. Various properties of the solutions of (1)-(3) are obtained through those of $\mathfrak{U}$.

1. The evolution equation in $H^{1}(0,1)$. In this section we derive an evolution equation (5) that is equivalent in $H^{1}(0,1)$ to the equation (1) with the boundary condition (3).

We begin by introducing some necessary spaces and operators. For each nonnegative integer $k, H^{k}$ denotes the Sobolev space $H^{k}(0,1)$ with inner product $(\cdot, \cdot)_{k}$ and norm $|\cdot|_{k}$. In particular, $H^{0}$ is the Lebesgue space $L^{2} \equiv L^{2}(0,1)$. The following closed subspace of $H^{1}$ plays an important role:

$$
H_{0}^{1}=\left\{v \in H^{1}: v(0)=v(1)=0\right\} .
$$

We shall use the fact that any element $v$ in $H^{1}$ can be identified with an absolutely continuous function on $[0,1]$ and satisfies $\sup _{x}|v(x)| \leq 3|v|_{1}$.

By $D$ we denote the differential operator $d / d x$ in $L^{2}$ with domain $H^{1}$. The operator $D$ is skew-symmetric in the sense that

$$
(D v, w)_{0}=-(v, D w)_{0}
$$

holds for $[v, w] \in H^{1} \times H_{0}^{1}$. In order to treat the nonlinear term on equation (1) we employ the composition operator $\Phi$ defined by $[\Phi v](x)=\phi(v(x))$ for $x \in[0,1]$ and $v \in H^{1}$. The operator $\Phi$ maps $H^{1}$ into itself and is locally Lipschitz continuous with respect to the $L^{2}$-norm in the sense that

$$
\left|\Phi v_{1}-\Phi v_{2}\right|_{0} \leq M_{r}\left|v_{1}-v_{2}\right|_{0}
$$

for $v_{i} \in H^{1}$ with $\left|v_{i}\right|_{1} \leq r, i=1,2$ and $r>0$, where

$$
M_{r}=\sup \left\{\left|\phi^{\prime}(\xi)\right|:|\xi| \leq 3 r\right\}, \quad r>0 .
$$


Finally, in addition to the sets $K(t), t \in[0, T]$, we define $K^{\prime}(t) \equiv\{w \in$ $\left.H^{2}: w(0)=h_{0}^{\prime}(t), w(1)=h_{1}^{\prime}(t)\right\}$ for $t \in[0, T]$. In view of (3) the set $K(0)$ is understood to be the class of initial data.

We now give a notion of (weak) solution of (1)-(3). A function $u(t, x)$ on $[0, T] \times$ $[0,1]$ is said to be a (weak) solution of (1)-(3) if the mapping $t \rightarrow u(t) \equiv u(t, \cdot)$, viewed as an $H^{1}$-valued function on $[0, T]$, satisfies $u(0)=u_{0}, u(t) \in K(t)$ for $t \in[0, T], u(\cdot) \in C^{1}\left([0, T] ; H^{1}\right)$ and

$$
\left(u^{\prime}(t), w\right)_{1}+(D \Phi u(t), w)_{0}=0 \quad \text { for } t \in(0, T) \text { and } w \in H_{0}^{1},
$$

where $u^{\prime}(t)$ stands for the strong derivative in $H^{1}$ of $u(t)$. Equation (1.4) is a generalized form of Equation (1) and this observation leads us to

DEFINITION 1.1. For each $t \in[0, T]$ we define an operator $A(t)$ by setting $w=A(t) v$ if $v \in K(t)$ and $w$ is a solution of the boundary value problem

$$
w^{\prime \prime}-w=(\phi(v))^{\prime} ; \quad w(0)=h_{0}^{\prime}(t), \quad w(1)=h_{1}^{\prime}(t) .
$$

Each of $A(t)$ is well-defined as a single-valued operator from $K(t)$ into $H^{2}$ since (1.5) has a unique solution $w$ in $H^{2}$ which is represented as

$$
w(x)=h_{0}^{\prime}(t) p(x)+h_{1}^{\prime}(t) q(x)+\int_{0}^{1} K(x, \xi) \phi(v(\xi)) d \xi,
$$

where $x, \xi \in[0,1]$,

$$
\begin{gathered}
p(x)=\left(e^{2-x}-e^{x}\right) /\left(e^{2}-1\right), \quad q(x)=\left(e^{1+x}-e^{1-x}\right) /\left(e^{2}-1\right), \\
K(x, \xi)=\left[-e^{x+\xi}-\operatorname{sgn}(x-\xi) e^{|x-\xi|}+e^{2-(x+\xi)}+\operatorname{sgn}(x-\xi) e^{2-|x-\xi|}\right] / 2\left(e^{2}-1\right) .
\end{gathered}
$$

Formula (1.6) gives an integral representation of the operator $A(t)$.

By means of the operators defined above, the problem (1)-(3) is reduced to the Cauchy problem (5), (7) in the following sense:

PROPOSITION 1.2. An $H^{1}$-valued function $u(\cdot)$ on $[0, T]$ is a $C^{1}$-solution of (5) iff $u(0)=u_{0}, u(\cdot) \in C^{1}\left([0, T] ; H^{1}\right), u(t) \in K(t), u^{\prime}(t) \in K^{\prime}(t)$ for $t \in[0, T]$, and (1.4) holds for $t \in(0, T)$ and $w \in H_{0}^{1}$.

2. Properties of the operators $A(t)$. We begin by defining an $H^{1}$-valued function $g(\cdot)$ on $[0, T]$ by

$$
[g(t)](x)=h_{0}(t) p(x)+h_{1}(t) q(x) \quad \text { for } t \in[0, T] \text { and } x \in[0,1] .
$$

As is easily seen, $g(\cdot) \in C^{1}\left([0, T] ; H^{1}\right)$ and $\left[g^{\prime}(t)\right](x)=h_{0}^{\prime}(t) p(x)+h_{1}^{\prime}(t) q(x)$ for $t \in[0, T]$ and $x \in[0,1]$. Now the operators $A(t), t \in[0, T]$, have two remarkable properties listed below.

LEMMA 2.1. (i) $|A(s) v-A(t) w|_{1} \leq|\Phi v-\Phi w|_{0}+\left|g^{\prime}(s)-g^{\prime}(t)\right|_{1}$ for $s, t \in[0, T]$, $v \in K(s)$ and $w \in K(t)$.

(ii) There are continuous functions $\alpha, \beta, \gamma:[0, T] \rightarrow[0, \infty)$ such that

$$
\left(A(t) v-g^{\prime}(t), v-g(t)\right)_{1} \leq \alpha(t)|v-g(t)|_{1}^{2}+\beta(t)|v-g(t)|_{1}+\gamma(t)
$$

for $t \in[0, T]$ and $v \in K(t)$.

PRoOF. (i) Let $s, t \in[0, T], v \in K(s)$ and $w \in K(t)$. If $r \in[0, T]$ and $z \in K(r)$, then $A(r) z=D^{2} A(r) z-D \Phi z$ and $A(r) z-g^{\prime}(r) \in H_{0}^{1}$. Also, $\left(I-D^{2}\right) g^{\prime}(r)=0$ for 
$r \in[0, T]$. Hence the application of (1.1) yields

$$
\begin{aligned}
\left|A(s) v-A(t) w-g^{\prime}(s)+g^{\prime}(t)\right|_{1}^{2} & =\left(\Phi v-\Phi w, D\left[A(s) v-A(t) w-g^{\prime}(s)+g^{\prime}(t)\right]\right)_{0} \\
& \leq|\Phi v-\Phi w|_{0}\left|D\left[A(s) v-A(t) w-g^{\prime}(s)+g^{\prime}(t)\right]\right|_{0} .
\end{aligned}
$$

From this estimate Assertion (i) follows.

(ii) In view of the growth condition (4) one finds nonnegative numbers $a, b, c$ such that $|\phi(\xi)| \leq a|\xi|^{2}+b|\xi|+c$ for $\xi \in \mathbf{R}$. Let $w=A(t) v$. Noting that $w=D^{2} w-D \Phi v$, $g^{\prime}(t)=D^{2} g^{\prime}(t)$ and $v-g(t) \in H_{0}^{1}$, we have

$$
\begin{aligned}
(w- & \left.g^{\prime}(t), v-g(t)\right)_{1}=(\Phi v, D(v-g(t)))_{0}=\int_{h_{0}(t)}^{h_{1}(t)} \phi(\xi) d \xi-(\Phi v, D g(t))_{0} \\
\leq & \left(a \cdot \max _{x}|[D g(t)](x)|\right)|v-g(t)|_{0}^{2}+\left(a\left|D g^{2}(t)\right|_{0}+b|D g(t)|_{0}\right)|v-g(t)|_{0} \\
& +\left(a\left|g^{2}(t)\right|_{0}+b|g(t)|_{0}+c\right)|D g(t)|_{0}+\left|\int_{h_{0}(t)}^{h_{1}(t)} \phi(\xi) d \xi\right|,
\end{aligned}
$$

where $g^{2}(\cdot)$ denotes the $H^{1}$-valued function on $[0, T]$ defined by $\left[g^{2}(t)\right](x)=$ $[g(t)](x)^{2}$. Hence the desired functions $\alpha, \beta, \gamma$ are obtained as follows:

$$
\begin{aligned}
& \alpha(t)=a \cdot \max _{x}|[D g(t)](x)|, \quad \beta(t)=a\left|D g^{2}(t)\right|_{0}+b|D g(t)|_{0}, \\
& \gamma(t)=\left(a\left|g^{2}(t)\right|_{0}+b|g(t)|_{0}+c\right)|D g(t)|_{0}+\left|\int_{h_{0}(t)}^{h_{1}(t)} \phi(\xi) d \xi\right| \cdot \quad \text { Q.E.D. }
\end{aligned}
$$

REMARK 2.2. The choice of the numbers $a, b, c$ depends upon the nonlinearity of $\phi$ : If $\phi(\xi)=\xi^{2} / 2+\xi$, then $a=1 / 2, b=1$ and $c=0$. If $\phi$ is Lipschitz continuous, then $a=0, b=\operatorname{Lip}(\phi)$ and $c=0$. Further, if $\phi$ is bounded, then $a=b=0$ and $c=\sup |\phi(\xi)|$. We do not know whether or not Assertion (ii) is still valid without the growth condition (4).

REMARK 2.3. Assertion (i) of Lemma 2.1 implies the following facts:

(a) Although $K(s) \cap K(t)=\varnothing$ for $s, t \in[0, T]$ with $h_{i}(s) \neq h_{i}(t)$, the mapping $(t, v) \rightarrow A(t) v$ is continuous in the sense that $t_{n} \rightarrow t$ in $[0, T], v_{n} \in K\left(t_{n}\right)$, and $v_{n} \rightarrow v \in K(t)$ in $H^{1}$ imply $A\left(t_{n}\right) v_{n} \rightarrow A(t) v$ in $H^{1}$.

(b) For each $t \in[0, T]$, the operator $A(t)$ is Lipschitz continuous on the intersection of $K(t)$ and the closed ball $B_{r}(0)=\left\{v \in H^{1}:|v|_{1} \leq r\right\}$ for each $r>0$.

LEMMA 2.4. Given an $r>0$ there exists a constant $\lambda_{0}=\lambda_{0}(r)>0$ such that for $t \in[0, T), \lambda \in\left(0, \lambda_{0}\right) \cap(0, T-t]$ and $v \in K(t)$ with $|v|_{1} \leq r$ one finds an element $v_{\lambda} \in K(t+\lambda)$ with the following properties:

$$
\begin{aligned}
& v_{\lambda}-\lambda A(t+\lambda) v_{\lambda}=v+g(t+\lambda)-g(t)-\lambda g^{\prime}(t+\lambda), \\
& (1-\lambda \alpha(t+\lambda)) \max \left\{1,\left|v_{\lambda}-g(t+\lambda)\right|_{1}\right\} \\
& \quad \leq \max \left\{1,|v-g(t)|_{1}\right\}+\lambda(\beta(t+\lambda)+\gamma(t+\lambda)) .
\end{aligned}
$$

PROOF. We begin by defining a nonnegative nondecreasing function $\rho(\cdot)$ on $[0, T]$ by $\rho(\xi)=\sup \left\{\left|g^{\prime}(s)-g^{\prime}(t)\right|_{1}: s, t \in[0, T],|s-t| \leq \xi\right\}$ for $\xi \in[0, T]$. Then $\lim _{\xi \downarrow 0} \rho(\xi)=0$ since $g^{\prime}(\cdot) \in C\left([0, T] ; H^{1}\right)$. Let $t \in[0, T), r>0$ and let $v \in$ 
$K(t) \cap B_{r}(0)$. Choose any $\lambda_{1}>0$ so that $\rho(\xi) \leq 1$ for $\xi \in\left(0, \lambda_{1}\right)$, set $R \equiv r+2$, and define $\lambda_{0}=\lambda_{0}(r)$ by

$$
\lambda_{0}=\min \left\{\lambda_{1}, 1 /\left(R M_{R}+\sup \left|g^{\prime}(t)\right|_{1}\right)\right\} .
$$

Let $\lambda \in\left(0, \lambda_{0}\right)$ and set $w=v+g(t+\lambda)-g(t)-\lambda g^{\prime}(t+\lambda)$. We show that $w$ belongs to the range $R(I-\lambda A(t+\lambda))$ of the operator $I-\lambda A(t+\lambda)$. To this end we define a mapping $\Gamma: K(t+\lambda) \cap B_{R}(0) \rightarrow H^{1}$ by $\Gamma z=w+\lambda A(t+\lambda) z$ for $z \in K(t+\lambda) \cap B_{R}(0)$. It is easy to see that the mapping $\Gamma$ maps $K(t+\lambda)$ into itself. Next we see in a way similar to the proof of Lemma 2.1(i) that $|A(s) z|_{1} \leq|\Phi z|_{0}+\left|g^{\prime}(s)\right|_{1}$ for $s \in[0, T]$ and $z \in K(s)$. Hence we infer from (1.2), (2.2) and the definition of $R$ that

$$
\begin{aligned}
|\Gamma z|_{1} & \leq|w|_{1}+\lambda|A(t+\lambda) z|_{1} \leq|w|_{1}+\lambda|\Phi z|_{0}+\lambda\left|g^{\prime}(t+\lambda)\right|_{1} \\
& \leq r+\rho(\lambda)+\lambda_{0}\left[R M_{R}+\sup \left|g^{\prime}(t)\right|_{1}\right] \leq R
\end{aligned}
$$

provided $z \in K(t+\lambda) \cap B_{R}(0)$. Moreover Lemma 2.1(i) and (1.2) together imply that $|\Gamma z-\Gamma \hat{z}|_{1} \leq \lambda M_{R}|z-\hat{z}|_{0}$ for $z, \hat{z} \in K(t+\lambda) \cap B_{R}(0)$. Therefore $\Gamma$ has a unique fixed point $v_{\lambda}$ in $K(t+\lambda) \cap B_{R}(0)$ and

$$
v+g(t+\lambda)-g(t)-\lambda g^{\prime}(t+\lambda)=w=v_{\lambda}-\lambda A(t+\lambda) v_{\lambda}
$$

which proves Assertion (i).

To show (ii) we recall that

$$
\begin{aligned}
& \left(A(t+\lambda) v_{\lambda}-g^{\prime}(t+\lambda), v_{\lambda}-g(t+\lambda)\right)_{1} \\
& \quad \leq \alpha(t+\lambda)\left|v_{\lambda}-g(t+\lambda)\right|_{1}^{2}+\beta(t+\lambda)\left|v_{\lambda}-g(t+\lambda)\right|_{1}+\gamma(t+\lambda)
\end{aligned}
$$

by Lemma 2.1 (ii). Using this relation we have

$$
\begin{aligned}
\left|v_{\lambda}-g(t+\lambda)\right|_{1}^{2}= & \left(v-g(t)+\lambda A(t+\lambda) v_{\lambda}-\lambda g^{\prime}(t+\lambda), v_{\lambda}-g(t+\lambda)\right)_{1} \\
\leq & |v-g(t)|_{1}\left|v_{\lambda}-g(t+\lambda)\right|_{1}+\lambda \alpha(t+\lambda)\left|v_{\lambda}-g(t+\lambda)\right|_{1}^{2} \\
& +\lambda \beta(t+\lambda)\left|v_{\lambda}-g(t+\lambda)\right|_{1}+\lambda \gamma(t+\lambda),
\end{aligned}
$$

from which we obtain Assertion (ii). Q.E.D.

REMARK 2.5. Lemma 2.4 states that the operators $A(t), t \in[0, T]$, satisfy the "tangency condition"

$$
\lim _{\lambda \downarrow 0} \lambda^{-1} d(v, R(I-\lambda A(t+\lambda)))=0 \quad \text { for } v \in K(t) .
$$

However the domain $K(t)$ of $A(t)$ is not necessarily contained in $R(I-\lambda A(t+\lambda))$. In fact, suppose $z-\lambda A(t+\lambda) z=v$ and $A(t+\lambda) z=w$. Then $z(0)=h_{0}(t+\lambda)$, $z(1)=h_{1}(t+\lambda), w(0)=h_{0}^{\prime}(t+\lambda)$ and $w(1)=h_{1}^{\prime}(t+\lambda)$, and hence we would have the relations $h_{i}(t+\lambda)-\lambda h_{i}^{\prime}(t+\lambda)=h_{i}(t), i=0,1$. But this is not the case in general. 
3. The evolution operator $\mathfrak{U}$. Applying Lemmas 2.1 and 2.4 , we obtain

THEOREM 3.1. There exists a nonlinear evolution operator $\mathfrak{U}=\{U(t, s): 0 \leq$ $s \leq t \leq T\}$ constrained in $\mathfrak{K}=\{K(s): 0 \leq s \leq T\}$ with the following properties:

(a) $U(t, s) z=z+\int_{s}^{t} A(\tau) U(\tau, s) z d \tau$ for $0 \leq s \leq t \leq T$ and $z \in K(s)$.

(b) $|U(t, s) z-g(t)|_{1} \leq \exp \left(\int_{s}^{t} \alpha(\tau) d \tau\right)\left[\max \left\{1,|z-g(s)|_{1}\right\}+\int_{s}^{t}(\beta(\tau)+\gamma(\tau)) d \tau\right]$ for $0 \leq s \leq t \leq T$ and $z \in K(s)$.

(c) Let $s \in[0, T), r>0$, and let

$$
R=\exp \left(\int_{0}^{T} \alpha(\tau) d \tau\right)\left[r+1+2 \sup |g(t)|_{1}+\int_{0}^{T}(\beta(\tau)+\gamma(\tau)) d \tau\right] .
$$

Then $|U(t, s) z-U(t, s) \hat{z}|_{k} \leq \exp \left(M_{R}(t-s)\right)|z-\hat{z}|_{k}$ for $z, \hat{z} \in K(s) \cap B_{r}(0), t \in[s, T]$ and $k=0,1$, where $M_{R}$ is defined by (1.3).

(d) For $u_{0} \in K(0)$, the $H^{1}$-valued function $u(t) \equiv U(t, 0) u_{0}$ on $[0, T]$ is a unique solution of the problem (1)-(3).

PROOF. Let $s \in[0, T), r_{0}>0$ and $z \in K(s) \cap B_{r_{0}}(0)$. Let $r \in \mathbf{R}$ satisfy $r \geq \exp (2 T \cdot \sup \alpha(t))\left[r_{0}+1+2 \sup |g(t)|_{1}+T \cdot \sup (\beta(t)+\gamma(t))\right]$, and define $\lambda_{0}$ by (2.2). Let $n_{0}$ be such that $(T-s) / n_{0}<\min \left\{\lambda_{0}, 1 / 2 \sup \alpha(t)\right\}$ and set $h_{n}=(T-s) / n$ and $t_{j}^{n}=s+j h_{n}$ for $j, n$ with $0 \leq j \leq n$ and $n \geq n_{0}$. Then Lemma 2.4 ensures that for each $n \geq n_{0}$ one finds a finite sequence $\left(z_{j}^{n}\right)_{j=0}^{n}$ in $H^{1}$ such that $z_{0}^{n}=z$, $z_{j}^{n} \in K\left(t_{j}^{n}\right)$,

$$
z_{j}^{n}-h_{n} A\left(t_{j}^{n}\right) z_{j}^{n}=z_{j-1}^{n}+g\left(t_{j}^{n}\right)-g\left(t_{j-1}^{n}\right)-h_{n} g^{\prime}\left(t_{j}^{n}\right),
$$

$$
\begin{aligned}
\mid z_{j}^{n}- & \left.g\left(t_{j}^{n}\right)\right|_{1} \\
& \leq \prod_{i=1}^{j}\left(1-h_{n} \alpha\left(t_{i}^{n}\right)\right)^{-1}\left[\max \left\{1,|z-g(s)|_{1}\right\}+\sum_{i=1}^{j} h_{n}\left(\beta\left(t_{i}^{n}\right)+\gamma\left(t_{i}^{n}\right)\right)\right]
\end{aligned}
$$
for $1 \leq j \leq n$. Since we have an a priori estimate $(3.2)$ as well as $\left|z_{j}^{n}\right|_{1} \leq$
$\exp (2 T \cdot \sup \alpha(t))\left[|z|_{1}+1+2 \sup |g(t)|_{1}+T \cdot \sup (\beta(t)+\gamma(t))\right] \leq r$, one can proceed with an induction argument to obtain the finite sequence as mentioned above. Set $\varepsilon_{j}^{n}=h_{n}^{-1}\left(g\left(t_{j}^{n}\right)-g\left(t_{j-1}^{n}\right)\right)-g^{\prime}\left(t_{j}^{n}\right)$. Then (3.1) can be written as.

$$
z_{j}^{n}-z_{j-1}^{n}=h_{n}\left[A\left(t_{j}^{n}\right) z_{j}^{n}+\varepsilon_{j}^{n}\right], \quad j=1,2, \ldots, n ; z_{0}^{n}=z .
$$

The form (3.3) is regarded as an approximating discrete scheme for (5), and each $\varepsilon_{j}^{n}$ is understood to be an error term at the $j$ th step and is estimated as

$$
\left|\varepsilon_{j}^{n}\right|_{1} \leq \int_{0}^{1}\left|g^{\prime}\left(\theta t_{j}^{n}+(1-\theta) t_{j-1^{\prime}}^{n}\right)-g^{\prime}\left(t_{j}^{n}\right)\right|_{1} d \theta \leq \rho\left(h_{n}\right),
$$

where $\rho$ is the function defined at the beginning of the proof of Lemma 2.4. Hence $\lim _{j \rightarrow \infty} \sup _{1 \leq j \leq n}\left|\varepsilon_{j}^{n}\right|_{1}=0$, and the convergence theorem due to Kobayasi, Kobayashi and Oharu [7, Theorem 3.5] can be applied to conclude that there is an $H^{1}$-valued, continuous function $u(t ; s, z)$ on $[s, T]$ and that $z_{j}^{n}$ converges to $u(t ; s, z)$ as $n \rightarrow \infty$ and $t_{j}^{n} \rightarrow t \in[s, T]$. Since $z_{j}^{n} \in K\left(t_{j}^{n}\right)$ for $1 \leq j \leq n$ and $n \geq 1$, it is seen that $u(t ; s, z) \in K(t)$ for $t \in[s, T]$. We now define a family $\mathfrak{U}$ of operators $U(t, z)$, $0 \leq s \leq t \leq T$, by

$$
U(t, s) z=u(t ; s, z) \quad \text { for } s, t \in[0, T] \text { with } s \leq t \text { and } z \in K(s) .
$$


It can be shown without difficulty (see Theorem 4.1 of $[\mathbf{7}]$ ) that the family $\mathfrak{U}$ forms a nonlinear evolution operator constrained in the family $\mathfrak{K}$. In view of (3.3) and Lemma 2.1(i) we see that $u(t ; s, z)=z+\int_{s}^{t} A(\tau) u(\tau ; s, z) d \tau$ for $t \in[s, T]$, from which the first assertion (a) follows. Assertion (b) is obtained by using (3.2). Assertion (c) is deduced from (a), (b), Lemma 2.1(i) and (1.2). Assertion (d) is a consequence of (a) and Proposition 1.2. Q.E.D.

REMARK 3.2. In the above proof one can also apply a result of Pavel [11, Theorem 3.1] instead of $\left[\mathbf{7}\right.$, Theorem 3.5]. Let $u_{0} \in K(0)$ and set $u(t, x)=\left[U(t, 0) u_{0}\right](x)$ for $(t, x) \in[0, T] \times[0,1]$. Then Assertion (a) and the integral representation (1.6) together imply that $u$ satisfies the integral equation

$$
\begin{aligned}
u(t, x)= & u_{0}(x)+\left(h_{0}(t)-h_{0}(0)\right) p(x)+\left(h_{1}(t)-h_{1}(0)\right) q(x) \\
& +\int_{0}^{t} \int_{0}^{1} K(x, \xi) \phi(u(\tau, \xi)) d \xi d \tau
\end{aligned}
$$

for $(t, x) \in[0, T] \times[0,1]$. From this it follows that if $u_{0} \in C^{2}[0,1]$, then $u$ satisfies equation (1) on all of $(0, T) \times(0,1)$. Further regularity properties of $u$ are obtained through this integral equation (cf. $[\mathbf{3}, \mathbf{6}])$.

REMARK 3.3. As is seen from Remark 2.5 and the proof of Theorem 3.1, it is required to employ an approximating discrete scheme (3.3) which includes "error terms". The above evolution operator $\mathfrak{U}$ gives an example of nonlinear evolution operators defined on noncylindrical domains. In comparison with the evolution operators discussed in $[\mathbf{4}, \mathbf{5}, \mathbf{8}, \mathbf{1 0}]$, this suggests the usefulness of the theory of evolution operators with time-dependent domains.

\section{REFERENCES}

1. T. Benjamin, J. Bona and J. Mahony, Model equations for long waves in nonlinear dispersive systems, Philos. Trans. Roy. Soc. London Ser. A 272 (1972), 47-78.

2. J. Bona and P. Bryant, A mathematical model for long waves generated by wavemakers in nonlinear dispersive systems, Proc. Cambridge Philos. Soc. 73 (1973), 391-405.

3. J. Bona and V. Dougalis, An initial- and boundary-value problem for a model equation for propagation of long waves, J. Math. Anal. Appl. 75 (1980), 503-522.

4. M. Crandall and A. Pazy, Nonlinear evolution equations in Banach spaces, Israel J. Math. 11 (1972), 57-94.

5. L. Evans, Nonlinear evolution equations in an arbitrary Banach space, Israel J. Math. 26 (1977), 1-42.

6. T. Iwamiya, S. Oharu and T. Takahashi, On the semigroup approach to some nonlinear dispersive equations, Lecture Notes in Numer. Appl. Anal., vol. 1, Kinokuniya Book Store Co., Tokyo, 1979, pp. 95-134.

7. K. Kobayasi, Y. Kobayashi and S. Oharu, Nonlinear evolution operators in Banach spaces, Osaka J. Math. 21 (1984), 281-310.

8. , Nonlinear evolution operators in Banach spaces. II, Hiroshima Math. J. (to appear).

9. L. Medeiros and M. Miranda, Weak solutions for a nonlinear dispersive equation, J. Math. Anal. Appl. 59 (1977), 432-441.

10. R. E. Showalter, Sobolev equations for nonlinear dispersive systems, Applicable Anal. 7 (1978), 297-308.

11. N. Pavel, Nonlinear evolution equations governed by $f$-quasi-dissipative operators, Nonlinear Anal. 5 (1981), 449-468.

Department of Mathematics, Hiroshima University, Hiroshima, Japan

National Aerospace Laboratory, Chofu, Tokyo, Japan 